\title{
The Phylogeny of the Genus Clostridium: Proposal of Five New Genera and Eleven New Species Combinations
}

\author{
M. D. COLLINS, ${ }^{1}$ P. A. LAWSON,${ }^{1 *}$ A. WILLEMS,${ }^{1}$ J. J. CORDOBA,${ }^{1}$ J. FERNANDEZ-GARAYZABAL, ${ }^{1}$ \\ P. GARCIA, ${ }^{1}{ }^{\text {J. CAI }}{ }^{1}$ H. HIPPE, ${ }^{2}$ AND J. A. E. FARROW ${ }^{1}$ \\ Institute of Food Research, Department of Microbiology, Earley Gate, Reading RG6 2EF, United Kingdom, ${ }^{1}$ and \\ Deutsche Sammlung von Mikroorganismen und Zellkulturen, D-38124 Braunschweig, Germany ${ }^{2}$
}

\begin{abstract}
The 16S rRNA gene sequences of 34 named and unnamed clostridial strains were determined by PCR direct sequencing and were compared with more than 80 previously determined clostridial sequences and the previously published sequences of representative species of other low- $\mathbf{G}+\mathrm{C}$-content gram-positive genera, thereby providing an almost complete picture of the genealogical interrelationships of the clostridia. The results of our phylogenetic analysis corroborate and extend previous findings in showing that the genus Clostridium is extremely heterogeneous, with many species phylogenetically intermixed with other sporeforming and non-spore-forming genera. The genus Clostridium is clearly in need of major revision, and the rRNA structures defined in this and previous studies may provide a sound basis for future taxonomic restructuring. The problems and different possibilities for restructuring are discussed in light of the phenotypic and phylogenetic data, and a possible hierarchical structure for the clostridia and their close relatives is presented. On the basis of phenotypic criteria and the results of phylogenetic analyses the following five new genera and 11 new combinations are proposed: Caloramator gen. nov., with Caloramator fervidus comb. nov.; Filifactor gen. nov., with Filifactor villosus comb. nov.; Moorella gen. nov., with Moorella thermoacetica comb. nov. and Moorella thermoautotrophica comb. nov.; Oxobacter gen. nov., with Oxobacter pfennigii comb. nov.; Oxalophagus gen. nov., with Oxalophagus oxalicus comb. nov.; Eubacterium barkeri comb. nov.; Paenibacillus durum comb. nov.; Thermoanaerobacter kivui comb. nov.; Thermoanaerobacter thermocopriae comb. nov.; and Thermoanerobacterium thermosaccharolyticum comb. nov.
\end{abstract}

Great advances have been made over the past decade in unravelling the phylogenetic complexities within the grampositive endospore-forming bacteria. For example, $16 \mathrm{~S}$ rRNA oligonucleotide cataloging and, more recently, almost complete rRNA (or gene) sequencing have shown that the aerobic endospore-forming bacilli are phylogenetically very heterogeneous, consisting of at least six highly divergent lines $(1,11$, 51). As a result of these studies, taxonomic reorganization of the genus Bacillus was initiated with the introduction of the genus Alicyclobacillus for some acidophilic species (51) and the genus Paenibacillus (2) for Bacillus polymyxa and its close relatives (rRNA group 3 [1]). Although the remainder of the genus is still in need of taxonomic revision, the phylogenetic groups established by rRNA analysis are already forming the foundation for a new molecular data-based taxonomy for this group of organisms. Knowledge of the natural interrelationships within the anaerobic genus Clostridium is more fragmented than knowledge of the interrelationships among the aerobic bacilli. The earliest, and until recently the most comprehensive, phylogenetic study of the genus Clostridium was published by Johnson and Francis (22), who demonstrated that there is considerable diversity within the genus by using DNA-rRNA pairing methods but unfortunately did not include nonclostridial gram-positive reference organisms. It was only with the advent of oligonucleotide cataloging that the very considerable phylogenetic incoherence of the genus Clostridium was realized $(15,32,46,47)$. During the $1980 \mathrm{~s}$ the oligonucleotide catalogs of more than 30 clostridia were determined (see reference 43 for a review), and members of the

* Corresponding author. Mailing address: Institute of Food Research, Reading Laboratory, Earley Gate, Whiteknights Road, Reading RG6 2EF, United Kingdom. Phone: (0734) 357226. Fax: (0734) 267917. genus were shown to belong to several deeply branching lineages, some of which also include nonclostridial species.

Although 16S rRNA oligonucleotide cataloging has without question provided valuable insights into the phylogenetic interrelationships of the clostridia and their relatives, it is now recognized that full rRNA sequences provide far greater precision for constructing phylogenetic trees (52). If phylogenetic data are to be used as the basis for future taxonomic restructuring of the genus Clostridium, it is imperative that as many species as possible be sequenced and that the branching patterns of trees be resolved with confidence. In recent years there has been considerable progress toward this end, with complete (or nearly complete) 16S rRNA sequences available for more than 80 clostridial species $(5,19,20,27,29,30,34,35$, $37,38,50)$. In this paper we describe the $16 \mathrm{~S}$ rRNA gene sequences of an additional 34 clostridial strains and the results of a comparative sequence analysis and thereby provide an almost complete picture of the genealogical interrelationships in the genus. The results of our phylogenetic analysis are discussed in the context of a possible future taxonomic rearrangement of the genus.

\section{MATERIALS AND METHODS}

Cultures. With the exception of the Clostridium tetani strain (National Collection of Type Cultures strain NCTC 279) and the Clostridium rectum strain (National Collection of Industrial and Marine Bacteria strain NCIMB $10651^{\mathrm{T}}[\mathrm{T}=$ type strain]), all clostridial strains were obtained from the Deutsche Sammlung von Mikroorganismen und Zellkulturen. Details concerning the strains which we examined are shown in Table 1, and the strains were cultivated as recommended in the culture collection catalogs.

16S rRNA gene sequence determination. Genomic DNA was 
TABLE 1. Clostridium strains examined and their 16S rRNA accession numbers

\begin{tabular}{|c|c|c|}
\hline Species & Strain $^{a}$ & EMBL accession no. \\
\hline C. absonum & DSM $599^{\mathrm{T}}$ & X77842 \\
\hline C. aerotolerans & DSM $5434^{\mathrm{T}}$ & $\mathrm{X} 76163$ \\
\hline "C. aminobutyricum" & DSM 2634 & $X 76161$ \\
\hline "C. caliptrosponum" & DSM 5905 & X77843 \\
\hline C. celatum & DSM $1785^{\mathrm{T}}$ & $\times 77844$ \\
\hline C. colinum & DSM $6011^{\mathrm{T}}$ & X76748 \\
\hline "C. corinoforum" & DSM 5906 & X76742 \\
\hline C. durum & DSM $1735^{\mathrm{T}}$ & X77846 \\
\hline "C. favososponum" & DSM 5907 & $\times 76749$ \\
\hline C. felsineum & DSM $794^{\mathrm{T}}$ & X77851 \\
\hline "C. filamentosum" & DSM 6645 & X77847 \\
\hline C. formicoaceticum & DSM $92^{\mathrm{T}}$ & X77836 \\
\hline C. glycolicum & DSM $1288^{\mathrm{T}}$ & $\times 76750$ \\
\hline C. halophilum & DSM $5387^{\mathrm{T}}$ & X77837 \\
\hline C. hastiforme & DSM $5675^{\mathrm{T}}$ & X77848 \\
\hline C. homopropionicum & DSM $5847^{\mathrm{T}}$ & X76744 \\
\hline C. intestinalis & DSM $6191^{\mathrm{T}}$ & X76740 \\
\hline "C. kainantoi" & DSM 523 & X77834 \\
\hline C. lentocellum & DSM $5427^{\mathrm{T}}$ & $\mathrm{X} 76162$ \\
\hline C. litorale & DSM $5388^{\mathrm{T}}$ & X77845 \\
\hline "C. longisporum" & DSM 8431 & X76164 \\
\hline C. magnum & DSM $2767^{\mathrm{T}}$ & X77835 \\
\hline "C. neopropionicum" & DSM $3847^{\mathrm{T}}$ & $\times 76746$ \\
\hline C. oxalicum & DSM $5503^{\mathrm{T}}$ & $\mathrm{X} 77840$ \\
\hline C. pfennigii & DSM $3222^{\mathrm{T}}$ & X77838 \\
\hline C. polysaccharolyticum & DSM $1801^{\mathrm{T}}$ & X77839 \\
\hline C. propionicum & DSM $1682^{T}$ & X77841 \\
\hline "C. quinii" & DSM $6736^{\mathrm{T}}$ & X76745 \\
\hline C. rectum & NCIMB $10651^{\mathrm{T}}$ & $\mathrm{X} 77850$ \\
\hline Clostridium sp. & DSM 6877 & X76747 \\
\hline Clostridium sp. & BN11 & X75909 \\
\hline C. tetani & NCTC $279^{\mathrm{T}}$ & $\times 74770$ \\
\hline "C. thermoamylolyticum" & DSM 2335 & X76743 \\
\hline C. xylanolyticum & DSM $6555^{\mathrm{T}}$ & X76739 \\
\hline
\end{tabular}

${ }^{a}$ DSM, Deutsche Sammlung von Mikroorganismen und Zellkulturen; NCIMB, National Collection of Industrial and Marine Bacteria; NCTC, National Collection of Type Cultures.

extracted from cells in the mid-logarithmic growth phase and was purified by the method of Lawson et al. (26). 16S rRNA gene fragments were generated by PCR as previously described (19). Amplified products were purified by using a Magic DNA Clean-up System (Promega) and were sequenced directly by using $\alpha-{ }^{35}$ S-labeled dATP and a Sequenase version 2.0 sequencing kit (United States Biochemicals) (19).

Analysis of sequence data. The $16 \mathrm{~S}$ rRNA gene sequences which we determined and the sequences of other clostridia and reference strains (obtained from the EMBL Data Library and the Ribosomal Database Project) were aligned by using programs in the Wisconsin Molecular Biology Package (7) and were analyzed by the distance matrix method of Fitch and Margoliash (13) and the neighbor-joining method of Saitou and Nei (39), using a VAX computer. The stability of relationships was assessed by using the programs SEQBOOT, DNADIST, NEIGHBOR, and CONSENSE of the PHYLIP package (12). A minimum of 1,000 bootstrap trees were generated for each data set.

Nucleotide sequence accession numbers. The 16S rRNA gene sequences which we determined have been deposited in the EMBL Data Library under the accession numbers shown in Table 1.

\section{RESULTS AND DISCUSSION}

The almost complete 16S rRNA sequences of 34 strains of clostridia were determined by direct sequencing of PCRamplified rRNA gene products. These new sequences were aligned and compared with homologous sequences of more than 200 other clostridia and selected reference strains belonging to low-G $+\mathrm{C}$-content gram-positive genera available from EMBL and the Ribosomal Database Project. Because of incomplete sequence data for some reference strains, and also to eliminate possible errors due to the extremely variable V1 region, approximately 100 nucleotides at the $5^{\prime}$ end of the rRNA were omitted from the alignments. Levels of sequence similarity for an approximately 1,330-nucleotide region (ranging from position 101 to position 1410 in the Escherichia coli numbering system) were calculated, and derived distances were used to determine the phylogenetic interrelationships of clostridia and other low-G+C-content taxa. The significance of the branching order of trees was estimated by bootstrapping. A complete matrix of the levels of sequence similarity which we determined is available upon request from P. A. Lawson.

The results of the treeing programs confirmed and extended the results of previous comparative rRNA studies and demonstrated the marked phylogenetic incoherence of the genus Clostridium. From our analyses it was evident that many clostridia formed very deep, but nevertheless distinct, clusters (Fig. 1 and 2). Almost one-half of the clostridial species, including Clostridium butyricum, the type species of the genus, belonged to a phylogenetically well-defined cluster (designated cluster I). The detailed phylogenetic interrelationships within this cluster are shown in Fig. 1. The remaining clostridial species exhibited very considerable degrees of phylogenetic diversity and formed numerous clusters and individual lines of descent. Two species, Clostridium durum and Clostridium oxalicum, were found to be phylogenetically remotely related to other clostridial species and were recovered within the confines of a large supercluster which encompassed members of the genus Bacillus and their non-spore-forming relatives (e.g., the genera Staphylococcus and Listeria). Figure 2 is a tree which shows the full genealogical complexities of the clostridia and their close relatives. Brief descriptions of the taxonomic compositions of the various clusters and sublines are given below.

Cluster I. Cluster I is the largest of the clostridial groups and is equivalent to rRNA group I of Johnson and Francis (22). Of the 34 new strains sequenced, 13 were found to be members of this cluster. Several of these strains exhibited specific affinities with other species or species groups within cluster I. For example, Clostridium absonum was closely related to Clostridium baratii, while the invalid vesicular cap-forming species "Clostridium caliptrosporum" (strain DSM 5905), which was isolated from soil (24), was highly related to Clostridium acetobutylicum and Clostridium beijerinckii (Fig. 1). Two other vesicular cap-forming species, "Clostridium corinoforum" (strain DSM 5906) and "Clostridium favososporum" (strain DSM 5907), which were also isolated from soil (24), were found to be almost identical to each other (level of similarity, $99.9 \%$ ) and were highly related to the pectinolytic amylolytic species Clostridium puniceum (level of similarity, 99.5\%). Clearly, chromosomal DNA-DNA hybridization data will be necessary to determine whether the vesicular cap-forming organisms and their close relatives ( $C$. acetobutylicum, $C$. beijerinckii, and $C$. puniceum) warrant separate species status. A similar problem of species identification was encountered with the bison rumen organism "Clostridium longisporum" 


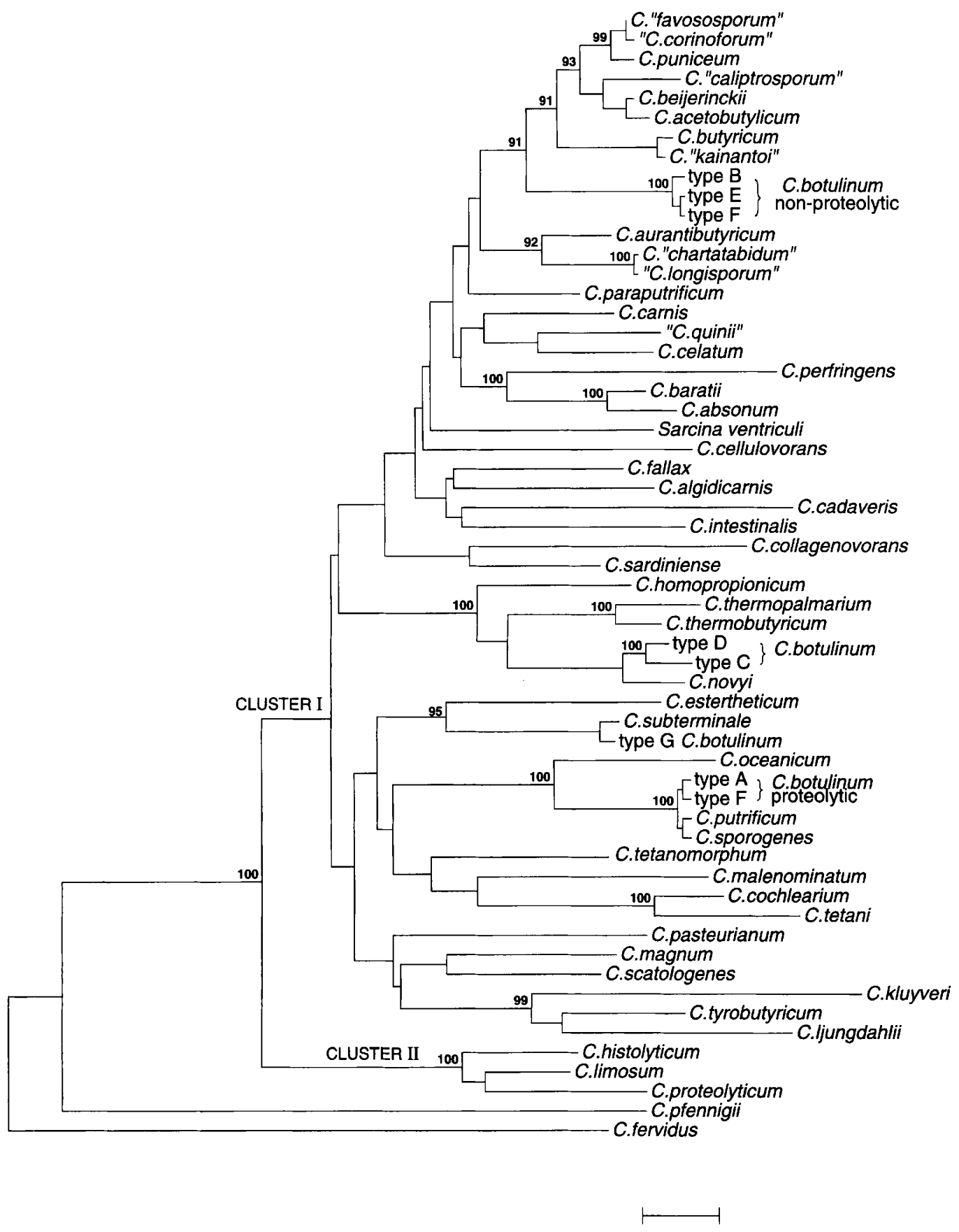

FIG. 1. Dendogram showing the interrelationships within cluster I (Clostridium group I) and closely related species. The tree was constructed by using the neighbor-joining method and bootstrap values calculated from 1,000 trees. Bar $=1 \%$ sequence divergence.

DSM 8431, which exhibited 99.9\% 16S rRNA sequence similarity with the ovine rumen species "Clostridium chartatabidum" (23). C. tetani NCTC 279 was significantly related to Clostridium cochlearium (level of similarity, 97.5\%). The recovery of $C$. tetani in cluster I was not expected as this species was placed in a quite separate group (rRNA homology group II; equivalent to cluster XI in this study) by Johnson and Francis (22). A second strain of C. tetani (NCTC 5404), which originally was isolated from a case of human tetanus, was also examined (unpublished data) and was found to be identical to the type strain, thereby confirming its placement in cluster I.
Clostridium homopropionicum surprisingly exhibited high levels of sequence relatedness ( $>95 \%$ ) with Clostridium novyi, Clostridium botulinum types $\mathrm{C}$ and $\mathrm{D}$, and the thermophilic species Clostridium thermobutyricum and Clostridium thermopalmarium. The results of bootstrap calculations (bootstrap value, 100) reinforced the significance of this subgroup (Fig. 1). In a previous study Rainey et al. (38) demonstrated that $C$. thermobutyricum and $C$. thermopalmarium were members of Clostridium group $\mathrm{I}$, although a close association with $C$. botulinum types $\mathrm{C}$ and $\mathrm{D}$ and their relatives was not reported by these authors. Clostridium celatum, Clostridium magnum, 


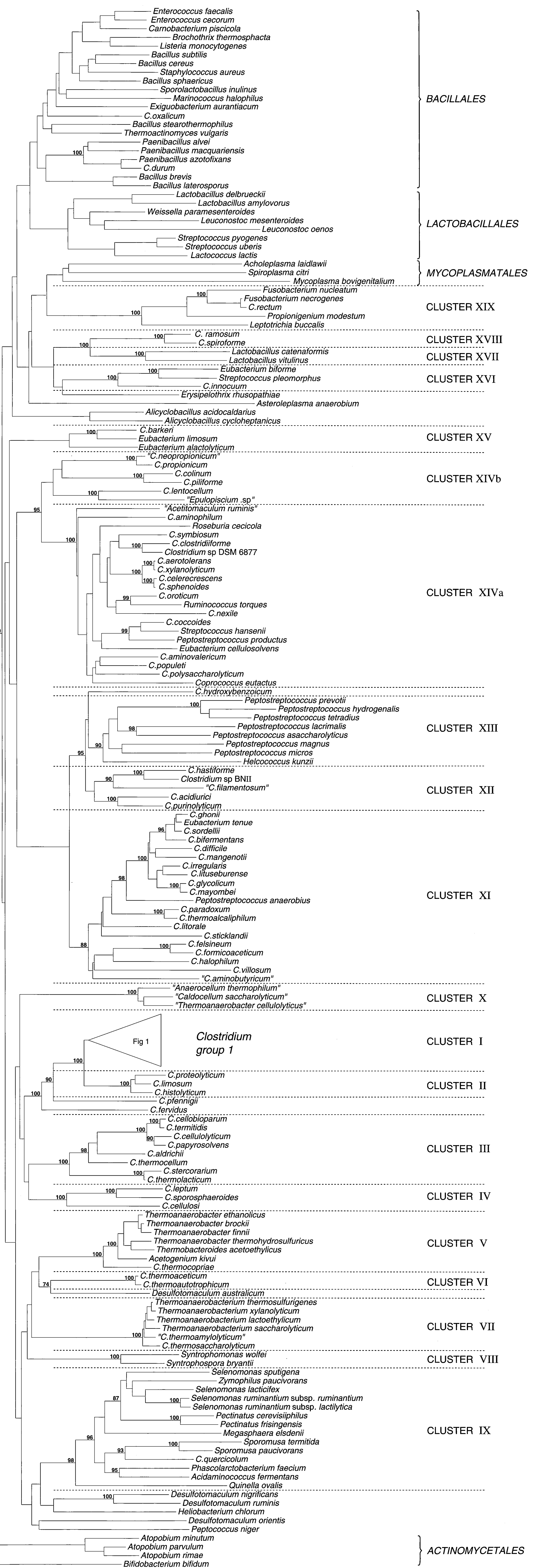


and Clostridium intestinalis also grouped in cluster I but exhibited only loose associations with other species (Fig. 1).

As shown in previous studies, Clostridium group I of Johnson and Francis (22) is a phylogenetically distinct group $(3,27$, $37,50)$. Members of this group exhibit relatively high levels of intracluster similarity (generally $>90 \%$ ) despite having markedly different phenotypes (for example, the group includes saccharolytic and proteolytic species, as well as psychrophiles, mesophiles, and thermophiles). In this study this group was found to be well separated from other clostridial clusters or lines irrespective of the treeing programs employed. Since group I contains the type species, $C$. butyricum, it is now generally accepted that the genus Clostridium should be retained for organisms belonging to this group.

Cluster II. As shown previously (27), cluster II is composed of three species (Clostridium histolyticum, Clostridium limosum, and Clostridium proteolyticum) and represents a distinct line at the periphery of cluster I. Species belonging to this group exhibit levels of sequence similarity of $>96 \%$ with each other but are significantly less closely related (levels of similarity, generally $<92 \%$ ) to cluster I species. Phenotypically, $C$. histolyticum, C. limosum, and C. proteolyticum resemble each other in producing acetate as the major end product of metabolism and in being highly proteolytic. These species probably constitute a natural group worthy of generic status, although clear phenotypic separation from a redefined genus Clostridium (cluster I) may prove to be problematical.

Cluster III. Cluster III consists of eight cellulolytic species and corresponds to group $\mathrm{E}$ of Rainey et al. (38). The levels of intracluster similarity ranged from 87 to $99 \%$. Although cluster III contains both mesophiles and thermophiles and the members of this cluster differ considerably in their chromosomal DNA base compositions (range, approximately 28 to $42 \mathrm{~mol} \%$ $\mathrm{G}+\mathrm{C}$ ), this group was identified consistently irrespective of the type of analysis performed and was confirmed $(37,38)$ to be statistically significant (bootstrap value, 100).

Cluster IV. Consistent with the results of previous investigations (27), Clostridium leptum and Clostridium sporosphaeroides were found to be closely related (level of similarity, approximately $94 \%$; bootstrap value, 100), with Clostridium cellulosi exhibiting a more peripheral association (level of similarity, approximately $89 \%$; bootstrap value, 100). The members of cluster IV are phenotypically heterogeneous (e.g., this cluster includes both mesophiles and thermophiles) and exhibit a broad chromosomal DNA $\mathrm{G}+\mathrm{C}$ content range (approximately 27 to $52 \mathrm{~mol} \%$ ) (18). However, the results of bootstrap calculations indicate that the relationship among these species is significant, and, in contrast to the results of a previous study (38), the thermophile $C$. cellulosi did not exhibit any rearrangement when transversion analysis was used (data not shown). In terms of sequence divergence cluster IV probably represents a suprageneric or family group.

Cluster V. Rainey et al. (38) showed that Thermoanaerobacter species, Acetogenium kivui, and Clostridium thermocopriae constitute a phylogenetically coherent group (designated group A). The results of our analysis confirmed these findings. Despite some phenotypic differences, high sequence similarity values (approximately 93 to 99\%) and bootstrap calculations (bootstrap values, 100 ) clearly indicated that $A$. kivui and $C$. thermocopriae should be reclassified in the genus Thermoanaerobacter (38).

Cluster VI. Cluster VI consists of Clostridium thermoaceticum and Clostridium thermoautotrophicum and corresponds to group B of Rainey et al. (38). These homoacetogenic sporeforming species differ from other clostridia in their high DNA base compositions (approximately 53 to $55 \mathrm{~mol} \% \mathrm{G}+\mathrm{C}$ ) and in the presence of LL-diaminopimelic acid in their cell wall peptidoglycans and, as shown previously, form a distinct line worthy of genus status (38). Desulfotomaculum australicum was peripherally associated with this cluster.

Cluster VII. Cluster VII is equivalent to group C of Rainey et al. (38) and corresponds to the genus Thermoanaerobacterium. As shown by Rainey et al. (38), Clostridium thermosaccharolyticum is also a member of this group and is clearly misclassified. The invalid species "Clostridium thermoamylolyticum" (strain DSM 2335) exhibited approximately 98 to $99 \%$ sequence relatedness with Thermoanaerobacterium species. In view of these very high levels of sequence similarity, chromosomal DNA-DNA hybridization data will be necessary to determine whether this starch-degrading organism, which is able to grow at $\mathrm{pH} 3.5$, represents a distinct species within the genus Thermoanaerobacterium.

Cluster VIII. The fatty acid $\beta$-oxidizing species Syntrophomonas wolfei and Syntrophospora bryantii constitute cluster VIII. The genera Syntrophomonas and Syntrophospora have recently been assigned to a new family, the Syntrophomonadaceae (53). However, the level of evolutionary divergence (approximately 6\%) between the two species seems to be more consistent with classification as members of a genus than with classification as separate genera in a family.

Cluster IX. Cluster IX corresponds to the Sporomusa branch of the Clostridium phylum and, as shown previously $(8,27)$, includes a heterogeneous collection of spore-forming and non-spore-forming organisms, many of which are gram negative. Although this cluster exhibits considerable internal structure and overall depth (range of intracluster similarity values, approximately 80 to $99 \%$ ), bootstrap calculations (bootstrap value, 99) indicate that it is a phylogenetically significant group at the suprageneric level. Within this cluster, the taxa Sporomusa, Acidaminococcus, Pectinatus, Selenomonas, Megasphaera, Quinella, and Phascolarctobacterium are distinct genera. In contrast to the results of a previous study and despite a significantly lower reported chromosomal DNA base composition (41), Zymophilus paucivorans was found to be phylogenetically intermixed with Selenomonas species (Fig. 2). The phylogenetic separateness of the genera Selenomonas and Zymophilus clearly merits further investigation. Consistent with previous studies, Clostridium quercicolum exhibited a specific association with the genus Sporomusa (level of similarity, approximately 89 to $90 \%$ ). In terms of evolutionary divergence, $C$. quercicolum is as distant from the genus Sporomusa as, for example, the genera Phascolarctobacterium and Acidaminococcus are from each other (8) and should therefore probably be placed in a new genus.

Cluster X. Cluster X corresponds to group D of Rainey et al. (38) and consists of several highly saccharolytic spore-forming thermophiles ("Anaerocellum thermophilum," "Caldocellum saccharolyticum," "Thermoanerobacter cellulolyticus"), none of which have been validly described. High intracluster similarity values $(>95 \%)$ and bootstrap calculations (bootstrap value, 100) support the view that this group represents a distinct genus.

Cluster XI. Cluster XI, which includes Clostridium lituseburense and its relatives, is equivalent to rRNA homology group II-A of Johnson and Francis (22). Five new sequences were recovered within the confines of this group. Clostridium glycolicum was found to be genetically highly related to Clostridium mayombei (level of similarity, $>99 \%$ ), whereas Clostridium felsineum was found to be closely related to Clostridium formicoaceticum (level of similarity, approximately 97\%). The remaining two species, "Clostridium aminobutyricum" and Clostridium halophilum, formed relatively distinct lines. Cluster 
$\mathrm{XI}$ is taxonomically heterogeneous and, as noted previously (27), includes the non-spore-forming species Peptostreptococcus anaerobius and Eubacterium tenue. Despite the marked range of phenotypes exhibited by members of cluster XI, the group is phylogenetically well defined (bootstrap significance, 100). The relatively broad range of intracluster similarity values (approximately 85 to $99 \%$ ) indicates that cluster XI represents a suprageneric, possibly family, group. C. lituseburense and several other species (Clostridium bifermentans, Clostridium difficile, C. glycolicum, Clostridium ghonii, Clostridium irregularis, C. mayombei, Clostridium mangenotii, Clostridium sordellii, Eubacterium tenue) form a distinct subgroup (levels of similarity, $>93 \%$; bootstrap value, 100 ) within cluster $\mathrm{XI}$, as do $C$. felsineum and $C$. formicoaceticum and the recently described thermotolerant alkaliphiles Clostridium paradoxum and Clostridium thermoalcaliphilum $(29,30)$. These three subclusters clearly represent new genera. All of the remaining cluster XI species form relatively distinct sublines exhibiting no specific associations.

Cluster XII. Cluster XII is phenotypically incoherent and is a loose association of four clostridial species and an unnamed non-spore-forming bacterium (strain BN11) that was isolated from a wastewater pond of a sugar refinery and is used as a source of a potent creatinine deiminase (16). Within this cluster, Clostridium acidiurici and Clostridium purinolyticum were specifically related (level of similarity, approximately 94\%; bootstrap value, 100) and probably belong to a single genus. The recovery of Clostridium hastiforme in cluster XII was completely unexpected. Although $C$. hastiforme has been shown to be distinct from $C$. botulinum type $\mathrm{G}$ and Clostridium subterminale on the basis of DNA-DNA pairing results (45), because of the phenotypic resemblance of these organisms it has generally been assumed that the relationship among them may be similar to the relationship which exists between $C$. botulinum group I and Clostridium sporogenes. However, $C$. botulinum type $G$ and $C$. subterminale form a distinct subline within cluster I and are phylogenetically remote from $C$. hastiforme. Even more remarkable was the highly significant association (level of similarity, approximately $95 \%$ ) between $C$. hastiforme and the unidentified Clostridium sp. (strain BN11). The latter organism contains a cell wall based on ornithine (type A4 $\beta$ ) and does not produce spores! The remaining species in cluster XII, "Clostridium filamentosum," exhibited only a loose association with other organisms and clearly constitutes a distinct subline.

Cluster XIII. As shown by Ezaki and colleagues (10) Peptostreptococcus asaccharolyticus, "Peptostreptococcus hydrogenalis," "Peptostreptococcus lacrimalis," Peptostreptococcus micros, Peptostreptococcus prevotii, and Peptostreptococcus tetradius form a natural group. The results of our study indicate that Helcococcus kunzii and possibly Clostridium hydroxybenzoicum are peripherally associated with this group. "Peptostreptococcus hydrogenalis," Peptostreptococcus prevotii, and Peptostreptococcus tetradius are specifically related to each other (levels of similarity, $>92 \%$ ), whereas the remaining peptostreptococcal species and $H$. kunzii form relatively long individual lines (levels of similarity, generally $<87 \%$ ) and probably constitute separate genera. It seems likely that this natural grouping of peptostreptococci and $H$. kunzii is at a suprageneric, possibly family, level.

Cluster XIV. Cluster XIV comprised more than 20 clostridial species, "Acetitomaculum ruminis," "Epulopiscium" sp., Ruminococcus torques, Coprococcus eutactus, Eubacterium cellulolyticum, Roseburia cecicola, and Streptococcus hansenii and exhibited very considerable phylogenetic depth (range of intracluster similarity values, approximately 80 to $99 \%$ ). With the exception of Clostridium aminovalericum $(33 \mathrm{~mol} \% \mathrm{G}+\mathrm{C})$ and Clostridium populeti $(28 \mathrm{~mol} \% \mathrm{G}+\mathrm{C})$ cluster XIV species are generally characterized by high $\mathrm{G}+\mathrm{C}$ contents (approximately 38 to $52 \mathrm{~mol} \%$ ).

Considerable internal structure was present within cluster XIV, and two major subgroups (designated subclusters XIVa and $\mathrm{XIVb}$ ) were readily discernable. Subcluster XIVa consisted of a phenotypically heterogeneous collection of organisms, including several non-spore-forming cocci (e.g., coprococci and ruminococci). Within subcluster XIVa, a biochemically unusual $N$-methylhydantoin-degrading mesophilic spore former (strain FS41 [= DSM 6877]) isolated from sewage sludge (17) was found to be specifically related to Clostridium clostridiiforme. This unnamed strain (17) phenotypically resembles $C$. clostridiiforme, but the observed level of sequence divergence (approximately $2.5 \%$ ) indicates that this organism represents a new species. Overall, the diverse phenotypes exhibited by members of subcluster XIVa, together with the wide range of observed evolutionary distances, indicate that this cluster is probably a suprageneric cluster. In this context, we believe that the recent assignment of Peptostreptococcus productus and Streptococcus hansenii to the genus Ruminococcus by Ezaki et al. (10) may prove to be premature. Several clostridial species (e.g., Clostridium oroticum) are more closely related to the ruminococcal species examined by Ezaki et al. (10) than Peptostreptococcus productus and Streptococcus hansenii are. The phylogenetic placement of Ruminococcus flavefaciens, the type species of the genus Ruminococcus, clearly needs to be resolved before taxonomic proposals for species of this subgroup can be made with confidence.

Subcluster XIVb consisted of a loose association of six species. Within this subcluster Clostridium propionicum was highly related to "Clostridium neopropionicum" (level of similarity, approximately $98 \%$ ), whereas the cellulolytic species Clostridium lentocellum exhibited a specific relationship with the "giant" nonculturable bacterium Epulopiscium sp. (level of similarity, $>90 \%$ ). Similarly, Clostridium colinum, which is associated with ulcerative enteritis of chickens, exhibited a highly significant affinity (level of similarity, approximately 95\%; bootstrap value, 100) with Clostridium piliforme, the noncultured causative agent of Tyzzer's disease (9). It is probable that these three sublines represent distinct genera.

Cluster XV. In agreement with previous studies $(27,51)$, Clostridium barkeri and Eubacterium limosum were found to be highly related on the basis of $16 \mathrm{~S}$ rRNA analysis data (level of similarity, approximately 95\%). Oligonucleotide cataloging data also indicated that the acetogen Acetobacterium woodii is a member of this group (47). Members of cluster XV possess an unusual type B cell wall peptidoglycan (47), have relatively high chromosomal DNA base compositions (approximately 38 to $45 \mathrm{~mol} \% \mathrm{G}+\mathrm{C}$ ), and clearly should be classified in a single genus.

Cluster XVI. Cluster XVI is a loose, albeit phylogenetically significant, association of three species (Clostridium innocuum, Eubacterium biforme, and Streptococcus pleomorphus) within the mycoplasma supercluster. Members of this cluster are incoherent with respect to cellular morphology, spore formation, and DNA base composition ( 32 to $44 \mathrm{~mol} \% \mathrm{G}+\mathrm{C}$ ). The relatively wide range of intracluster similarity values (approximately 88 to $93 \%$ ) indicates that the rank of this group is at a suprageneric, possibly family, level. $C$. innocuum is the most peripheral member of the group, and its level of sequence divergence (approximately 11 to $12 \%$ ) compared with the other two species indicates that it is a member of a separate genus. In contrast, the species Eubacterium biforme and Streptococcus pleomorphus are more highly related to each other 
(level of divergence, approximately 7\%), although whether these taxa represent a single genus or two closely related genera is currently unclear.

Cluster XVII. Lactobacillus catenaformis and Lactobacillus vitulinus clustered together, in accordance with the data of Weisburg et al. (48), and are phylogenetically far removed from the Lactobacillales. Although these organisms are undoubtedly related, the level of sequence divergence (approximately $10 \%$ ) is probably more consistent with classification as members of two closely related genera than classification as members of a single genus. $L$. catenaformis and $L$. vitulinus exhibited a loose, albeit significant, association with the species Clostridium spiroforme and Clostridium ramosum.

Cluster XVIII. As reported previously (27) C. spiroforme and $C$. ramosum belong to a natural group within a supercluster which includes mycoplasmas and their relatives. The very high level of sequence relatedness of $C$. spiroforme and $C$. ramosum (level of divergence, $<4 \%$ ), together with their phenotypic resemblance and similar DNA base compositions (26 to 27 mol\% $\mathrm{G}+\mathrm{C}$ ), indicates that these species should be reclassified in a single genus.

Cluster XIX. Propionigenium modestum and $C$. rectum form a distinct cluster with members of the genus Fusobacterium. The genus Propionigenium was described by Schink and Pfenning (40) to accomodate a novel gram-negative, non-sporeforming, propionate-producing, strictly anaerobic bacterium. Although this genus was originally assigned to the family Bacteroidaceae (40), the phylogenetic analysis clearly placed this taxon proximal to the periphery of the genus Fusobacterium in the Clostridium subphylum of the gram-positive bacteria. Evolutionary distance data indicate that the relationship between Propionigenium modestum and fusobacteria is at a family level rather than a genus level. The association of $C$. rectum with the genus Fusobacterium was unexpected, as $C$. rectum produces endospores. Despite this major phenotypic difference, $C$. rectum clusters well within the boundaries of the genus and exhibits a particularly close affinity with Fusobacterium necrogenes (level of sequence similarity, approximately $99 \%$ ). On the basis of phylogenetic findings $C$. rectum should be reclassified in the genus Fusobacterium, and the definition of the latter taxon should be emended to take into account the possibility of spore formation.

Individual lines. (i) Clostridium pfennigii. Bootstrap data indicate that $C$. pfennigii exhibits no significant affinity with any other species or group and belongs to a relatively long and isolated line. C. pfennigii exhibits some metabolic similarities to Acetobacterium woodii (25), but Acetobacterium woodii does not produce endospores and has a type B cell wall murein, and oligonucleotide cataloging $(46,47)$ has shown that it is phylogenetically closely related to $C$. barkeri and Eubacterium limosum (Fig. 2, cluster XV). In view of its isolated phylogenetic position and unique metabolism (it catabolyzes methoxybenzenoids to corresponding hydroxybenzenoids and butyrate, pyruvate to acetate and $\mathrm{CO}_{2}$, and $\mathrm{CO}$ to acetate and butyrate), C. pfennigii clearly merits a new genus.

(ii) Clostridium fervidus. As previously reported by Rainey et al. (38), C. fervidus exhibits a loose association with some other thermophilic species groups when normal evolutionary distances are used (clusters V to VII), while transversion analysis data (Fig. 2) indicate a peripheral association with cluster I and its relatives. However, despite some uncertainty with respect to these higher associations, $C$. fervidus clearly forms a distinct line at the genus level.

(iii) Clostridium durum. C. durum differs from most other clostridia in having a relatively high chromosomal DNA base composition $(50 \mathrm{~mol} \% \mathrm{G}+\mathrm{C})(42)$. Although $C$. durum is aerotolerant, this species was assigned to the genus Clostridium because it sporulates under anaerobic conditions (sporulation under aerobic conditions is a characteristic of the genus Bacillus) (42). It is evident from our data that $C$. durum is only remotely related to other clostridia, is phylogenetically a member of the genus Paenibacillus (rRNA group 3 bacilli [1, 2]), and is specifically related to Paenibacillus azotofixans (level of similarity, approximately $98 \%$ ).

(iv) Clostridium oxalicum. Although $C$. oxalicum is strictly anaerobic, the treeing programs showed that this organism is only remotely related to clostridia and consistently placed it within the boundaries of the supercluster encompassing the genus Bacillus and its aerobic and facultatively anaerobic relatives (Fig. 2). It is evident from both distance calculations (level of sequence similarity, generally $<87 \%$ ) and tree branching patterns that $C$. oxalicum represents a previously undescribed lineage. Phenotypically, $C$. oxalicum differs from all other spore-forming taxa in utilizing oxalate as a sole energy source and in its inability to utilize other organic acids, sugars, or alcohols (6). The highly unusual metabolism of $C$. oxalicum, together with its isolated phylogenetic position, indicates that this organism merits a new genus.

Taxonomic considerations. The genus Clostridium includes organisms which are obligately anaerobic, form endospores, are not able to carry out dissimilatory sulfate reduction, and possess a gram-positive type of wall structure. As a result of this simple definition, the genus Clostridium has become one of the largest genera of bacteria and presently contains more than 100 species. Although it has long been recognized that the genus is phenotypically heterogeneous, it is only with the advent of oligonucleotide cataloging (see reference 3 for a review of the early literature) and, more recently, 16S rRNA sequencing $(27,37,38)$ that the full phylogenetic diversity of the clostridia has become apparent. A remarkable result that has emerged from such studies is the phylogenetic intermixing of some clostridia with non-spore-forming organisms, challenging our perception of spore formation as an important criterion for determining relatedness. It is now clear that any future taxonomic rearrangement of the clostridia must include their non-spore-forming relatives and take into account phylogenetic relatedness.

Knowledge of the interrelationships of the genus Clostridium and its relatives is increasing so rapidly that a naturally based classification may be realized in the next few years. Considerable progress toward this goal would be made if the genus Clostridium were restricted to homology group I of Johnson and Francis (22). Despite considerable phenotypic diversity, numerous studies have shown that this group is phylogenetically distinct, and it is now generally recognized that homology group I should form the basis of a redefined genus Clostridium (50). However, the placement of Sarcina ventriculi, the type species of the genus Sarcina, in this group raises a serious nomenclatural problem, as the name Sarcina has priority (50). Even if the rules of nomenclatural priority were set aside, the inclusion of sporulating cocci in the genus Clostridium could present a major hurdle to deriving a phenotypically tight genus delineation. Restriction of the genus Clostridium to group I organisms would result in the remaining clostridial species losing their status as members of the genus, and they would require reclassification.

The results of previous studies and our analysis indicate that the non-group I clostridia represent a phylogenetically very broad range of organisms and form a plethora of lines and groups of various complexities and depths. Numerous phylogenetically significant associations can be discerned at higher (suprageneric) levels, indicating that a hierarchical system for 
these organisms may now be achievable. Fox et al. (15) outlined a possible hierarchy for gram-positive bacteria which was subsequently updated by Cato and Stackebrandt (3). An amended hierarchical structure for the clostridia and their relatives in light of current knowledge is shown in Table 2.

It now seems clear that any comprehensive taxonomic rearrangement of clostridia will result in a major proliferation of new genera. It is important to emphasize that this will not be due to overzealous taxonomic splitting but will be a consequence of the immense genealogical diversity of these organisms, which has become apparent only since molecular sequence data have been available. Some clostridial species form phylogenetically distinct groups with sufficient phenotypic coherence that they can be readily equated with genera. However, many other species form individual lines or sublines and exhibit only loose associations with other organisms. The taxonomic rank of many of these organisms is far more problematic. Although it is not possible to derive precise boundary conditions based on percentage $16 \mathrm{~S}$ rRNA sequence relatedness to delineate taxonomic rank, as an approximation most generic groupings exhibit a sequence divergence of $<6 \%$. When this guideline is used in conjunction with tree topology considerations (including confidence values), it seems likely that many of the lines and sublines will represent the nuclei of previously unrecognized genera. The considerable genealogical complexities in the suprageneric association designated cluster XI in this study are a case in point. This cluster contains a phylogenetically and phenotypically diverse assemblage of organisms. Within this cluster $C$. lituseburense and related species and two pairs of organisms, $C$. felsineum plus $C$. formicoaceticum and C. paradoxum plus $C$. thermoalcaliphilum, form three phylogenetically significant subgroups worthy of separate generic status. The evolutionary distances exhibited by most other members of cluster XI (e.g., Peptostreptococcus anaerobius, $C$. villosum, and $C$. aminobutyricum) are such that they too could be designated distinct genera with reasonable confidence. Not only is such a subdivision phylogenetically justifiable, but it would also reduce many phylogenetic and phenotypic inconsistencies. Subdivision into more manageable units would simplify the search for suitable characteristics to allow clear phenotypic circumscription and differentiation of new genera, which is recognized as a major obstacle $(18,27)$ in constructing a naturally based classification of the clostridia. Table 2 shows the lines and sublines which in our opinion represent new genera. This scheme will undoubtedly be modified and expanded as other clostridia and related organisms (e.g., the genus Eubacterium) are characterized. However, we are confident that this compilation of relationships established from the results of this and previous phylogenetic analyses will provide a firm basis for a comprehensive taxonomic restructuring of the clostridia and their low- $\mathrm{G}+\mathrm{C}$-content relatives in the near future.

It is not the purpose of this paper to formally propose a major taxonomic revision of the clostridia. However, some clostridial species are phylogenetically so distinct that they merit reclassification as separate genera, whereas others should be assigned to existing genera. Details of our proposals are given below.

Taxonomic proposals. (i) Description of Oxobacter gen. nov. Oxobacter (Ok.so.bac'ter. Gr. n. oxos, vinegar; Gr. hyp. masc. n. bakter, rod; M. L. Masc. n. Oxobacter, acetogenic rod). Cells are gram positive and rod shaped. Oval, subterminal to terminal spores are produced. Obligately anaerobic. Pyruvate is catabolized to acetate and $\mathrm{CO}_{2}, \mathrm{CO}$ is catabolized to acetate and butyrate, and methoxybenzenoids are catabolized to butyrate and hydroxybenzenoids. Other substrates, such as sug- ars, amino acids, organic acids, and alcohols, are not utilized as energy sources. The G+C content of the DNA is $38 \mathrm{~mol} \%$. The type species is Oxobacter pfennigii.

(ii) Description of Oxobacter pfennigii (Krumholz and Bryant) comb. nov. The description of Oxobacter pfennigii comb. nov. is identical to that proposed for $C$. pfennigii Krumholz and Bryant $1985,455^{\mathrm{AL}}(25)$. The type strain is DSM 3222.

(iii) Description of Oxalophagus gen. nov. Oxalophagus (Ok. sa.lo'pha.gus. Gr. n. oxalis, wood sorrel [from which the name of oxalic acid is derived]; Gr. masc. n. phagos, glutton; N. L. masc. n. Oxalophagus, oxalate eater). Cells are gram-positive, straight rods. Endospores are formed. The spores are oval and are located subterminally to centrally. Strictly anaerobic. Catalase negative; cytochromes are not produced. Oxalate and oxamate are decarboxylated to formate. Acetate is assimilated for cell carbon synthesis; no growth occurs with other organic acids, sugars, or alcohols. The $\mathrm{G}+\mathrm{C}$ content of the DNA is 35.4 to $37.2 \mathrm{~mol} \%$. The type species is Oxalophagus oxalicus.

(iv) Description of Oxalophagus oxalicus (Dehning and Schink) comb. nov. The description of Oxalophagus oxalicus comb. nov. is identical to that proposed for $C$. oxalicum Dehning and Schink $1989,83^{\mathrm{AL}}(6)$. The type strain is DSM 5503.

(v) Description of Caloramator gen. nov. Caloramator (Ca.lo ra.ma'tor. L. n. calor, heat; L. masc. n. amator, lover; N. L. masc. n. caloramator, heat lover). Cells are rod shaped and stain gram negative but are gram positive in wall structure. Endospores are produced; the endospores are spherical and subterminal to terminal and do not swell the sporangia. Obligately anaerobic. Glucose and some other sugars are fermented. The fermentation products from glucose are acetate, isobutyrate, isovalerate, valerate, lactate, and ethanol. Xylan is degraded. Cellulose is not degraded. Thermophilic (temperature range, approximately 37 to $80^{\circ} \mathrm{C}$ ). Unable to grow autotrophically or to produce sulfur from thiosulfate. The $\mathrm{G}+\mathrm{C}$ content of the DNA is $37 \mathrm{~mol} \%$. The type species is Caloramator fervidus.

(vi) Description of Caloramator fervidus (Patel, Monk, Littleworth, Morgan, and Daniel) comb. nov. The description of Caloramator fervidus comb. nov. is identical to that proposed for $C$. fervidus Patel, Monk, Littleworth, Morgan, and Daniel $1987,125^{\mathrm{AL}}$ (36). The type strain is ATCC 43204.

(vii) Description of Filifactor gen. nov. Filifactor (Fi.li.fac'tor. L. n. filum, thread; L. masc. n. factor, maker; N. L. masc. n. Filifactor, thread maker). Cells are long rods. Filaments may be formed. Cells in young cultures are gram positive; the Gram reaction is variable in old cultures. Spores are oval and subterminal. The products of fermentation are acetate, butyrate, isobutyrate, formate, and isovalerate. Pyruvate is converted to butyrate. The cell walls contain an ornithine-Dasparagine-type murein. The type species is Filifactor villosus.

(viii) Description of Filifactor villosus (Love, Jones, and Bailey) comb. nov. The description of Filifactor villosus comb. nov. is identical to that proposed for C. villosum Love, Jones, and Bailey $1979,241^{\mathrm{AL}}$ (31). The type strain is NCTC 11220.

(ix) Description of Moorella gen. nov. Moorella (Moo. rel'la. N. L. fem. n. Moorella, in honor of W. E. C. Moore, an American bacteriologist). Cells are rod shaped and occur singly, in pairs, or in chains. Cells are generally gram positive, although older cultures may stain gram negative. Spores are round to slightly oval and terminal or subterminal and distend the cells. Thermophilic. The optimum temperature for growth is 56 to $60^{\circ} \mathrm{C}$, and the maximum temperature for growth is 65 to $68^{\circ} \mathrm{C}$. Homoacetogenic, growing chemolithotrophically with $\mathrm{H}_{2}$ and $\mathrm{CO}_{2}$ or $\mathrm{CO}$, as well as chemoorganotrophically with some carbohydrates or methanol, producing acetate. Produces 
TABLE 2. Possible hierarchical structure for clostridia and their close relatives

Family 1: Clostridiaceae

Genus 1: Redefined genus Clostridium based on C. butyricum and its relatives (equivalent to group I of Johnson and Francis), including the genus Sarcina

Genus 2: C. histolyticum, C. limosum and C. proteolyticum

Genus 3: C. pfennigii

Genus 4: C. fervidus

Family 2: Thermoanaerobacteriaceae

Genus 1: Thermoanaerobacter

Genus 2: Thermoanaerobacterium

Genus 3: $C$. thermoaceticum and $C$. thermoautotrophicum

Genus 4: Desulfotomaculum australicum

Family 3

Genus 1: C. leptum and C. sporosphaeroides ${ }^{b}$

Genus 2: C. cellulosi

Family 4

Genus 1: $C$. stercorarium and $C$. thermolacticum

Genus 2: C. cellobioparum, C. cellulolyticum, C. papyrosolvens, C. termitidis, C. aldrichiic, and C. thermocellum ${ }^{c}$

Family 5: Syntrophomonadaceae

Genus 1: Syntrophomonas

Genus 2: Syntrophospora ${ }^{d}$

Family 6: Selenomonadaceae

Genus 1: Selenomonas

Genus 2: Acidaminococcus

Genus 3: Megasphaera

Genus 4: Pectinatus

Genus 5: Phascolarctobacterium

Genus 6: Quinella

Genus 7: Sporomusa

Genus 8: C. quercicolum

Genus 9: Zymophilus ${ }^{e}$

Family 7

Genus 1: "Anaerocellum thermophilum," "Caldocellum saccharolyticum," and "Thermoanaerobacter cellulolyticus"

Family 8: Ruminococcaceae

Genus 1: Ruminococcus

Genus 2: Coprococcus

Genus 3: Roseburia

Genus 4: "Acetitomaculum"

Genus 5: C. coccoides, Peptostreptococcus productus, and Streptococcus hansenii

Genus 6: Lachnospira

Genus 7: C. aminophilum

Uncertain status: C. aerotolerans, ${ }^{f} C$. aminovalericum, $C$. celerecrescens, C. populeti, $C$. polysaccharolyticum, C. sphenoides, C.xylanolyticum, ${ }^{f}$ C. clostridiiforme, C. nexile, C. symbiosum, Clostridium sp. strain DSM 6877, and Eubacterium cellulosolvens

Family 9: Fusobacteriaceae

Genus 1: Fusobacterium (including C. rectum) ${ }^{h}$

Genus 2: Propionigenium

Genus 3: Leptotrichia

Genus 4: Sebaldella

Family 10

Genus 1: "Epulopiscium" spp.

Genus 2: C. lentocellum

Genus 3: C. propionicum and "C. neopropionicum"

Genus 4: $C$. colinum and $C$. piliforme

Family 11: Eubacteriaceae

Genus 1: Eubacterium (Eubacterium limosum) and C. barkeri

Family 12

Genus 1: C. acidiurici and C. purinolyticum

Genus 2: $C$. hastiforme and Clostridium. sp. strain BN11

Genus 3: "C. filamentosum"

Family 13

Genus 1: Peptostreptococcus (Peptostreptococcus anaerobius)

Genus 2: C. bifermentans, $C$. difficile, C. irregularis, C. ghonii, C. glycolicum, C. lituseburense, C. mayombei, C. mangenotii, $C$. sordellii, and Eubacterium tenue

Genus 3: $C$. paradoxum and $C$. thermoalcaliphilum

Genus 4: $C$. sticklandii

Genus 5: C. villosum

Genus 6: "C. aminobutyricum"

Genus 7: C. formicoaceticum and C. felsineum

Genus 8: C. halophilum

Genus 9: C. litorale 
TABLE 2-Continued

Family 14: Helcococcaceae

Genus 1: Helcococcus

Genus 2: Peptostreptococcus micros

Genus 3: Peptostreptococcus tetradius, Peptostreptococcus prevotii, Peptostreptococcus vaginalis, Peptostreptococcus lactolyticus, and "Peptostreptococcus hydrogenalis"

Uncertain status: Peptostreptococcus asaccharolyticus, "Peptostreptococcus lacrimalis", Peptostreptococcus magnus, and C. hydroxybenzoicum

Distinct genera of uncertain higher affiliation

Genus 1: Desulfotomaculum (Desulfotomaculum nigrificans and Desulfotomaculum ruminis)

Genus 2: Heliobacterium

Genus 3: Peptococcus (Peptococcus niger)

Clostridia and relatives which are associated with members of the Mycoplasmatales

Genus 1: C. spiroforme and C. ramosum

Genus 2: L. catenaformis ${ }^{g}$ and $L$. vitulinus ${ }^{g}$

Genus 3: Eubacterium biforme and Streptococcus pleomorphus

Genus 4: C. innocuum

Genus 5: Erysipelothrix

${ }^{a}$ The position of $C$. fervidus is uncertain. When normal distances are used, this species is associated with some thermophilic species, while transversion analysis data indicate that it is peripherally associated with the group I clostridia and their relatives.

${ }^{b}$ It is not known whether these species are members of two closely related genera or a single genus.

${ }^{c}$ The significance of the association of these organisms with other species is unclear.

${ }^{d}$ The small evolutionary distance between the genera Syntrophomonas and Syntrophospora indicates that these taxa may represent a single genus.

${ }^{e}$ Whether this taxon can be separated from the genus Selenomonas is unclear.

${ }^{f}$ These organisms have almost identical sequences and may be members of the same species.

${ }^{8}$ These organisms are loosely associated with each other and may belong to separate genera.

${ }^{h}$ Although C. rectum forms endospores, it is clearly a member of the genus Fusobacterium.

a variety of electron carriers, including cytochrome $b$ and menaquinone MK-7. The cell walls contain LL-diaminopimelic acid and glycine. The $\mathrm{G}+\mathrm{C}$ content of the DNA is 53 to 55 mol\%. The type species is Moorella thermoacetica.

(x) Description of Moorella thermoacetica (Fontaine, Peterson, McCoy, and Johnson) comb. nov. The description of Moorella thermoacetica comb. nov. is identical to that proposed for $C$. thermoaceticum Fontaine, Peterson, McCoy, and Johnson $1942,707^{\mathrm{AL}}$ (14). The type strain is DSM 521.

(xi) Description of Moorella thermoautotrophica (Wiegel, Braun, and Gottschalk) comb. nov. The description of Moorella thermoautotrophica comb. nov. is identical to that proposed for $C$. thermoautotrophicum Wiegel, Braun, and Gottschalk 1982, 384 ${ }^{\mathrm{VP}}$ (49). The type strain is DSM 1974.

(xii) Description of Paenibacillus durum (Smith and Cato) comb. nov. The description of Paenibacillus durum comb. nov. is identical to that proposed for $C$. durum Smith and Cato $1974,1394^{\mathrm{AL}}$ (42). The type strain is ATCC 27763.

(xiii) Description of Eubacterium barkeri (Stadtman, Stadtman, Pastan, and Smith) comb. nov. The description of Eubacterium barkeri comb. nov. is identical to that proposed for C. barkeri Stadtman, Stadtman, Pastan, and Smith 1972, $760^{\mathrm{AL}}$ (45) and given in detail in Bergey's Manual of Systematic Bacteriology (4). The type strain is ATCC 25849.

(xiv) Description of Thermoanaerobacter kivui (Leigh and Wolfe) comb. nov. The description of Thermoanaerobacter kivui comb. nov. is identical to that proposed for Acetogenium kivui Leigh and Wolfe $1983,886^{\mathrm{AL}}$ (28). The type strain is ATCC 33488.

(xv) Description of Thermoanaerobacter thermocopriae (Jin, Yamasato, and Toda) comb. nov. The description of Thermoanaerobacter thermocopriae comb. nov. is identical to that proposed for C. thermocopriae Jin, Yamasato, and Toda 1988, $280^{\text {AL }}$ (21). The type strain is IAM 13577.

(xvi) Description of Thermoanaerobacterium thermosaccharolyticum (McClung) comb. nov. The description of Thermoanaerobacterium thermosaccharolyticum comb. nov. is identical to that proposed for C. thermosaccharolyticum McClung 1939, $200^{\mathrm{AL}}$ (33). The type strain is ATCC 7956.

\section{ACKNOWLEDGMENTS}

This work was supported by grants from the Ministry of Agriculture, Fisheries and Food and by grants BIOT-CT91-0294 and BIOT-CT930119) from the European Community.

\section{REFERENCES}

1. Ash, C., J. A. E. Farrow, S. Wallbanks, and M. D. Collins. 1991. Phylogenetic heterogeneity of the genus Bacillus revealed by comparative analysis of small-subunit ribosomal RNA sequences. Lett. Appl. Microbiol. 13:202-206.

2. Ash, C., F. G. Priest, and M. D. Collins. 1993. Molecular identification of rRNA group 3 bacilli (Ash, Farrow, Wallbanks and Collins) using a PCR probe test: proposal for the creation of a new genus Paenibacillus. Antonie van Leeuwenhoek 64:253-260.

3. Cato, E., and E. Stackebrandt. 1989. Taxonomy and phylogeny, p. 1-26. In N. P. Minton and D. J. Clark (ed.), Clostridia. Plenum Press, New York.

4. Cato, E. P., W. L. George, and S. M. Finegold. 1986. Genus Clostridium, p. 1141-1200. In P. H. A. Sneath, N. S. Mair, M. E. Sharpe, and J. G. Holt (ed.), Bergey's manual of systematic bacteriology, vol. 2. Williams and Wilkins, Baltimore.

5. Collins, M. D., U. M. Rodrigues, R. H. Dainty, R. A. Edwards, and T. A. Roberts. 1992. Taxonomic studies on a psychrophilic Clostridium from vacuum packed beef: description of Clostridium estertheticum sp. nov. FEMS Microbiol. Lett. 96:235-240.

6. Dehning, I., and B. Schink. 1989. Two new species of anaerobic oxalate-fermenting bacteria, Oxalobacter vibrioformis sp. nov. and Clostridium oxalicum sp. nov., from sediment samples. Arch. Microbiol. 153:79-84.

7. Devereux, J., P. Haeberli, and D. Smithies. 1984. A comprehensive set of sequence analysis programs for the VAX. Nucleic Acids Res. 12:387-395.

8. Dot, T. D., R. Osawa, and E. Stackebrandt. 1993. Phascolarctobacterium faecium gen. nov., spec. nov., a novel taxon of the Sporomusa group of bacteria. Syst. Appl. Microbiol. 16:380-384.

9. Duncan, A. J., R. J. Carman, G. L. Olsen, and K. H. Wilson. 1993. Assignment of the agent of Tyzzer's disease to Clostridium piliforme comb. nov. on the basis of $16 \mathrm{~S}$ rRNA sequence analysis. Int. J. Syst. Bacteriol. 43:314-318.

10. Ezaki, T., N. Li, Y. Hashimoto, H. Miura, and H. Yamamoto. 1994. 16 S ribosomal DNA sequences of anaerobic cocci and proposal of 
Ruminococcus hansenii comb. nov. and Ruminococcus productus comb. nov. Int. J. Syst. Bacteriol. 44:130-136.

11. Farrow, J. A. E., C. Ash, S. Wallbanks, and M. D. Collins. 1992. Phylogenetic analysis of the genera Planococcus, Marinococcus and Sporosarcina and their relationships to members of the genus Bacillus. FEMS Microbiol. Lett. 93:167-172.

12. Felsenstein, J. 1989. PHYLIP-phylogeny inference package (version 3.2). Cladistics 5:164-166.

13. Fitch, W. M., and E. Margoliash. 1967. Construction of phylogenetic trees: a method based on mutation distances as estimated from cytochrome c sequences is of general applicability. Science 155:279-284.

14. Fontaine, F. E., W. H. Peterson, E. McCoy, and M. J. Johnson. 1942. A new type of glucose fermentation by Clostridium thermoaceticum n. sp. J. Bacteriol. 43:701-715.

15. Fox, G. E., E. Stackebrandt, R. B. Hespell, J. Gibson, J. Maniloff, T. A. Dyer, R. S. Wolfe, W. E. Balch, R. S. Tanner, L. J. Magrum, L. B. Zablen, R. Blakemore, R. Gupta, L. Bonen, B. J. Lewis, D. A. Stahl, K. R. Luehrsen, K. Chen, and C. R. Woese. 1980. The phylogeny of prokaryotes. Science 209:457-463.

16. Gottschalk, E. M., H. Hippe, and F. Patzke. 1991. Creatinine deiminase (EC3. 4. 3. 21) from bacterium BNII: purification, properties and applicability in serum/urine creatinine assay. Clin. Chim. Acta 204:223-238.

17. Hermann, M., H. J. Knerr, N. Mai, A. Gross, and H. Kaltwasser. 1992. Creatinine and $\mathrm{N}$-methylhydantoin degradation in two newly isolated Clostridium species. Arch. Microbiol. 157:395-401.

18. Hippe, H., J. R. Andreesen, and G. Gottschalk. 1992. The genus Clostridium-nonmedical, p. 1800-1866. In A. Balows, H. G. Trüper, M. Dworkin, W. Harder, and K. H. Schleifer (ed.), The prokaryotes, vol. 2. Springer-Verlag, New York.

19. Hutson, R. A., D. E. Thompson, and M. D. Collins. 1993. Genetic interrelationships of saccharolytic Clostridium botulinum types B, $\mathrm{E}$ and $\mathrm{F}$ and related clostridia as revealed by small-subunit rRNA gene sequences. FEMS Microbiol. Lett. 108:103-110.

20. Hutson, R. A., D. E. Thompson, P. A. Lawson, R. P. SchockenItturino, E. C. Böttger, and M. D. Collins. 1993. Genetic interrelationships of proteolytic Clostridium botulinum types A, B, and F and other members of the Clostridium botulinum complex as revealed by small-subunit rRNA gene sequences. Antonie van Leeuwenhoek 64:278-283.

21. Jin, F., K. Yamasato, and K. Toda. 1988. Clostridium thermocopriae sp. nov., a cellulolytic thermophile from animal feces, compost, soil, and a hot spring in Japan. Int. J. Syst. Bacteriol. 38:279-281.

22. Johnson, J. L., and B. S. Francis. 1975. Taxonomy of the clostridia: ribosomal ribonucleic acid homologies among the species. J. Gen. Microbiol. 88:229-244.

23. Kelly, W. J., R. V. Asmundson, and D. H. Hopcroft. 1987. Isolation and characterization of a strictly anaerobic, cellulolytic spore former: Clostridium chartatabidum sp. nov. Arch. Microbiol. 147: 169-173.

24. Krasilnikov, N. A., G. E. Pivovarov, and V. I. Duda. 1971. Physiological properties of anaerobic soil bacteria which form vesicular caps on their spores. Microbiology (Engl. Transl. Mikrobiologiya) 40:783-788.

25. Krumholz, L. R., and M. P. Bryant. 1985. Clostridium pfennigii sp. nov. uses methoxyl groups of monobenzenoids and produces butyrate. Int. J. Syst. Bacteriol. 35:454-456.

26. Lawson, P. A., S. E. Gharbia, H. N. Shah, and D. R. Clark. 1989. Recognition of Fusobacterium nucleatum subgroups Fn-1, Fn-2 and $\mathrm{Fn}-3$ by ribosomal RNA gene restriction patterns. FEMS Microbiol. Lett. 65:41-46.

27. Lawson, P. A., P. L. Perez, R. A. Hutson, H. Hippe, and M. D. Collins. 1993. Towards a phylogeny of the clostridia based on $16 \mathrm{~S}$ rRNA sequences. FEMS Microbiol. Lett. 113:87-92.

28. Leigh, J. A., and R. S. Wolfe. 1983. Acetogenium kivui, a thermophilic acetogenic bacterium. Int. J. Syst. Bacteriol. 33:886.

29. Li, Y., M. Engle, N. Weiss, L. Mandelco, and J. Wiegel. 1994. Clostridium thermoalcaliphilum sp. nov., an anaerobic and thermotolerant facultative alkaliphile. Int. J. Syst. Bacteriol. 44:111-118.

30. Li, Y., L. Mandelco, and J. Wiegel. 1993. Isolation and characterization of a moderately thermophilic alkaliphile, Clostridium para- doxum sp. nov. Int. J. Syst. Bacteriol. 43:450-460.

31. Love, D. N., R. F. Jones, and M. Bailey. 1979. Clostridium villosum sp. nov. from subcutaneous abscesses in cats. Int. J. Syst. Bacteriol. 29:241-244.

32. Ludwig, W., M. Weizenegger, R. Kilpper-Bälz, and K. H. Schleifer. 1988. Phylogenetic relationships of anaerobic streptococci. Int. J. Syst. Bacteriol. 38:15-18.

33. McClung, L. S. 1935. Studies on anaerobic bacteria. IV. Taxonomy of cultures of a thermophilic species causing "swells" of canned food. J. Bacteriol. 29:189-202.

34. Olsen, G. J., R. Overbeek, N. Larsen, T. L. Marsh, M. J. McCaughey, M. A. Maciukenas, W. M. Kuan, T. J. Macke, Y. Xing, and C. R. Woese. 1992. The Ribosomal Database Project. Nucleic Acids Res. 20:2199-2200.

35. Paster, B. J., J. B. Russell, C. M. J. Yang, J. M. Chow, C. R. Woese, and R. Tanner. 1993. Phylogeny of the ammonia-producing ruminal bacteria Peptostreptococcus anaerobius, Clostridium sticklandii, and Clostridium aminophilum sp. nov. Int. J. Syst. Bacteriol. 43:107-110.

36. Patel, B. K. C., C. Monk, H. Littleworth, H. W. Morgan, and R. M. Daniel. 1987. Clostridium fervidus sp. nov., a new chemoorganotrophic acetogenic thermophile. Int. J. Syst. Bacteriol. 37:123-126.

37. Rainey, F. A., and E. Stackebrandt. 1993. 16S rRNA analysis reveals phylogenetic diversity among the polysaccharolytic clostridia. FEMS Microbiol. Lett. 113:125-128.

38. Rainey, F. A., N. L. Ward, H. W. Morgan, R. Toalster, and E. Stackebrandt. 1993. Phylogenetic analysis of anaerobic thermophilic bacteria: aid for their reclassification. J. Bacteriol. 175:47724779.

39. Saitou, N., and M. Nei. 1987. The neighbor-joining method: a new method for reconstructing phylogenetic trees. Mol. Biol. Evol. 4:406-425.

40. Schink, B., and N. Pfenning. 1982. Propionigenium modestum gen. nov., sp. nov., a new strictly anaerobic, nonsporing bacterium growing on succinate. Arch. Microbiol. 133:209-216.

41. Schleifer, K. H., M. Leuteritz, N. Weiss, W. Ludwig, G. Kirchhof, and H. S. Rüfer. 1990. Taxonomic study of anaerobic, gramnegative, rod-shaped bacteria from breweries: emended description of Pectinatus cerevisiiphilus and descriptions of Pectinatus frisingensis sp. nov., Selenomonas lacticifex $\mathrm{sp}$. nov., Zymophilus raffinosivorans gen. nov., sp. nov., and Zymophilus paucivorans sp. nov. Int. J. Syst. Bacteriol. 40:19-27.

42. Smith, L. D. S., and E. P. Cato. 1974. Clostridium durum sp. nov., the predominant organism in a sediment core from the Black Sea. Can. J. Microbiol. 20:1393-1397.

43. Stackebrandt, E. 1992. Unifying phylogeny and phenotypic diversity, p. 19-47. In A. Balows, H. G. Trüper, M. Dworkin, W. Harder, and K. H. Schleifer (ed.), The prokaryotes, vol. 1. Springer-Verlag, New York.

44. Stadtman, E. R., T. C. Stadtman, I. Pastan, and L. D. S. Smith. 1972. Clostridium barkeri sp. n. J. Bacteriol. 110:758-760.

45. Suen, J. C., C. L. Hatheway, A. G. Steigerwalt, and D. J. Brenner. 1988. Clostridium argentinense sp. nov.: a genetically homogeneous group composed of all strains of Clostridium botulinum toxin type $\mathrm{G}$ and some nontoxigenic strains previously identified as Clostridium subterminale and Clostridium hastiforme. Int. J. Syst. Bacteriol. 38:375-381.

46. Tanner, R. S., E. Stackebrandt, G. E. Fox, L. J. Gupta, L. J. Magrum, and C. R. Woese. 1982. A phylogenetic analysis of anaerobic eubacteria capable of synthesizing acetate from carbon dioxide. Curr. Microbiol. 7:127-132.

47. Tanner, R. S., E. Stackebrandt, G. E. Fox, and C. R. Woese. 1981. A phylogenetic analysis of Acetobacterium woodii, Clostridium barkeri, Clostridium butyricum, Clostridium lituseburense, Eubacterium limosum, and Eubacterium tenue. Curr. Microbiol. 5:35-38.

48. Weisburg, W. G., J. G. Tully, D. L. Rose, J. P. Petzel, H. Oyaizu, D. Yang, L. Mandelco, J. Sechrest, T. G. Lawrence, J. Van Etten, J. Maniloff, and C. R. Woese. 1989. A phylogenetic analysis of the mycoplasmas: basis for their classification. J. Bacteriol. 171:6455-6467.

49. Wiegel, J., M. Braun, and G. Gottschalk. 1981. Clostridium thermoautotrophicum species novum, a thermophile producing acetate from molecular hydrogen and carbon dioxide. Curr. Microbiol. 5:255-260. 
50. Willems, A., and M. D. Collins. 1994. Phylogenetic placement of Sarcina ventriculi and Sarcina maxima within group I Clostridium: a possible problem for the future revision of the genus Clostridium. Int. J. Syst. Bacteriol. 44:591-593.

51. Wisotzkey, J. D., P. Jurtshuk, Jr., G. E. Fox, G. Deinhard, and K. Poralla. 1992. Comparative sequence analysis of the 16S rRNA of Bacillus acidocaldarius, Bacillus acidoterrestris, and Bacillus cyclo- heptanicus: proposal for a new genus, Alicyclobacillus gen. nov. Int. J. Syst. Bacteriol. 42:263-269.

52. Woese, C. R. 1987. Bacterial evolution. Microbiol. Rev. 51:221-271.

53. Zhao, H., D. Yang, C. R. Woese, and M. P. Bryant. 1993. Assignment of fatty acid- $\beta$-oxidizing syntrophic bacteria to Syntrophomonadaceae fam. nov. on the basis of 16S rRNA sequence analysis. Int. J. Syst. Bacteriol. 43:278-286. 